\title{
Mass functions in coupled Dark Energy models.
}

\author{
Roberto Mainini, Silvio Bonometto \\ Department of Physics G. Occhialini - Milano-Bicocca University, \\ Piazza della Scienza 3, 20126 Milano, Italy and \\ I.N.F.N., Sezione di Milano
}

(Dated: October 1, 2018)

\begin{abstract}
We evaluate the mass function of virialized halos, by using Press \& Schechter (PS) and/or Steth \& Tormen (ST) expressions, for cosmologies where Dark Energy (DE) is due to a scalar selfinteracting field, coupled with Dark Matter (DM). We keep to coupled DE (cDE) models known to fit linear observables. To implement the PS-ST approach, we start from reviewing and extending the results of a previous work on the growth of a spherical top-hat fluctuation in cDE models, confirming their most intriguing astrophysical feature, i.e. a significant baryon-DM segregation, occurring well before the onset of any hydrodynamical effect. Accordingly, the predicted mass function depends on how halo masses are measured. For any option, however, the coupling causes a distortion of the mass function, still at $z=0$. Furthermore, the $z$-dependence of cDE mass functions is mostly displaced, in respect to $\Lambda \mathrm{CDM}$, in the opposite way of uncoupled dynamical DE. This is an aspect of the basic underlying result, that even a little DM-DE coupling induces relevant modifications in the non-linear evolution. Therefore, without causing great shifts in linear astrophysical observables, the DM-baryon segregation induced by the coupling can have an impact on a number of cosmological problems, e.g., galaxy satellite abundance, spiral disk formation, apparent baryon shortage, entropy input in clusters, etc..
\end{abstract}

PACS numbers: 98.80.-k, 98.65.-r 


\section{INTRODUCTION}

A first evidence of Dark Energy (DE) came from the Hubble diagram of SNIa, showing an accelerated cosmic expansion, but a flat cosmology with $\Omega_{m} \simeq 0.25, h \simeq 0.73$ and $\Omega_{b} \simeq 0.042$ is now required by CMB and LSS observations and this implies that the gap between $\Omega_{m}$ and unity is to be filled by a smooth non-particle component, whose nature is one of the main puzzles of cosmology. $\left(\Omega_{m, b}\right.$ : matter, baryon density parameters; $h$ : Hubble parameter in units of $100 \mathrm{~km} / \mathrm{s} / \mathrm{Mpc}$; CMB: cosmic microwave background; LSS: large scale structure.)

If $\mathrm{DE}$ is a false vacuum, its pressure/density ratio $w=p_{D E} / \rho_{D E}$ is strictly -1 . This option, however, implies a severe fine tuning at the end of the electroweak transition. Otherwise DE could be a scalar field $\phi$, self-interacting through a potential $V(\phi)$ 1], [2], so that

$$
\rho_{D E}=\rho_{k, D E}+\rho_{p, D E} \equiv \dot{\phi}^{2} / 2 a^{2}+V(\phi), \quad p_{D E}=\rho_{k, D E}-\rho_{p, D E},
$$

provided that dynamical equations yield $\rho_{k, D E} / V \ll 1 / 2$, so that $-1 / 3 \gg w>-1$. Here

$$
d s^{2}=a^{2}(\tau)\left(-d \tau^{2}+d x_{i} d x^{i}\right), \quad(i=1, . ., 3)
$$

is the background metric and dots indicate differentiation with respect to $\tau$ (conformal time). This kind of DE is dubbed dynamical DE (dDE) or quintessence; the $w$ ratio then exhibits a time dependence set by the shape of $V(\phi)$. Much work has been done on dDE (see, e.g., [3] and references therein), also aiming at restricting the range of acceptable $w(\tau)$ 's, so gaining an observational insight onto the physics responsible for the potential $V(\phi)$.

As a matter of fact, the dark cosmic components are one of the most compelling evidences of physics beyond the standard model of elementary interactions and, while lab experiments safely exclude non-gravitational baryon-DE interactions, DM-DE interactions are constrained just by cosmological observations. In turn, DM-DE interactions could ease the cosmic coincidence problem [4], i.e. that DM and DE densities, differing by orders of magnitude since ever, approach one another at today's eve.

In a number of papers, constraints on coupling, coming from CMB and LSS observations, were discussed [4, 5, 6]. This note aims at formulating predictions on the mass function of bound systems in coupled DE (cDE) cosmologies, by using Sheth \& Tormen 9] expressions, known to improve the original Press \& Schechter 8] approach. Our final scope amounts to finding stronger constraints on DM-DE coupling, arising from a comparison of observational 
data with our predictions, opening a basic window on the nature and origin of these very components. Our analysis will be restricted to SUGRA [10] and RP (Ratra-Peebles) 2] potentials

$$
\begin{array}{cr}
\text { SUGRA } & V(\phi)=\left(\Lambda^{\alpha+4} / \phi^{\alpha}\right) \exp \left(4 \pi \phi^{2} / m_{p}^{2}\right) \\
R P & V(\phi)=\left(\Lambda^{\alpha+4} / \phi^{\alpha}\right)
\end{array}
$$

( $m_{p}=G^{-1 / 2}$ : Planck mass), admitting tracker solutions. This will however enable us to focus on precise peculiarities, not caused by the shape of $V(\phi)$ but by the coupling itself. Let us also remind that, once the DE density parameter $\Omega_{D E}$ is assigned, either $\alpha$ or the energy scale $\Lambda$, in the potentials (3), can still be freely chosen. In this paper we show results for $\Lambda=10^{2} \mathrm{GeV}$; minor quantitative shifts occur when varying $\log _{10}(\Lambda / \mathrm{GeV})$ in the $1-4$ range. The RP potential will be mostly considered to test the effects of varying DE nature.

The effects of coupling can be seen in the background equations for DE and DM, reading

$$
\ddot{\phi}+2(\dot{a} / a) \dot{\phi}+a^{2} V_{, \phi}=\sqrt{16 \pi G / 3} \beta a^{2} \rho_{c}, \quad \dot{\rho}_{c}+3(\dot{a} / a) \rho_{c}=-\sqrt{16 \pi G / 3} \beta \rho_{c} \dot{\phi} ;
$$

here $\beta$ sets the coupling strength and, all through this paper, we take it constant (and, in particular, independent of $\phi$; a different case, which can be physically significant and may deserve a separate treatment [7]) with values $\beta=0.05$ or 0.20 . In previous work this was considered a small strength. CMB data set a limit $\beta<\sim 0.6$; a stronger constraint found by [6], by studying cluster profiles in n-body simulations, applies to RP potentials only.

On the contrary, even for the smaller coupling we considered, we find significant deviations from uncoupled models in non-linear observables. Such deviations exhibit intriguing features, suggesting possible ways out from a number of astrophysical problems, while the small coupling strength scarcely affects linear observables.

As far as the other model parameters are concerned, we take density parameters $\Omega_{m}=$ $0.25, \Omega_{b}=0.042, \Omega_{c}=0.208$ and $h=0.73$. This choice approaches the best fit of a $\Lambda$ CDM model to available data. A best fit to cDE models will certainly yield slightly different parameters, but this implies just minor shifts in our quantitative findings.

\section{BARYON AND DARK MATTER DYNAMICS}

The essential novel feature induced by DM-DE coupling in non-linear structures is baryon-DM segregation. This was shown in [1] (paper I, hereafter). The reason why segre- 
gation occurs can be easily illustrated by considering, aside of the eq. (44), ruling background dynamics, the couple of equations telling us how the baryon (DM) density fluctuations $\delta_{b}$ $\left(\delta_{c}\right)$ and velocity fields

$$
\theta_{c, b}=i \frac{\mathbf{k} \cdot \mathbf{v}_{\mathbf{c}, \mathbf{b}}}{\mathcal{H}}
$$

depend on $\tau(\mathcal{H}=\dot{a} / a ; \mathbf{k}$ : wavenumber of the fluctuation considered). They read

$$
\begin{aligned}
\delta_{c}{ }^{\prime \prime} & =-\delta_{c}{ }^{\prime}\left(1+\frac{\mathcal{H}^{\prime}}{\mathcal{H}}-2 \beta X\right)+\frac{3}{2}\left(1+\frac{4}{3} \beta^{2}\right) \Omega_{c} \delta_{c}+\frac{3}{2} \Omega_{b} \delta_{b}, \\
\delta_{b}{ }^{\prime \prime} & =-\delta_{b}{ }^{\prime}\left(1+\frac{\mathcal{H}^{\prime}}{\mathcal{H}}\right)+\frac{3}{2}\left(\Omega_{c} \delta_{c}+\Omega_{b} \delta_{b}\right), \\
\theta_{c}{ }^{\prime} & =-\theta_{c}\left(1+\frac{\mathcal{H}^{\prime}}{\mathcal{H}}-2 \beta X\right)-\frac{3}{2}\left(1+\frac{4}{3} \beta^{2}\right) \Omega_{c} \delta_{c}-\frac{3}{2} \Omega_{b} \delta_{b}, \\
\theta_{b}{ }^{\prime} & =-\theta_{b}\left(1+\frac{\mathcal{H}^{\prime}}{\mathcal{H}}\right)-\frac{3}{2}\left(\Omega_{c} \delta_{c}+\Omega_{b} \delta_{b}\right) .
\end{aligned}
$$

Here ${ }^{\prime}$ yields differentiation with respect to $\alpha=\ln a$ and

$$
X=\sqrt{4 \pi / 3} \dot{\phi} /\left(m_{p} \mathcal{H}\right)
$$

Both eqs. (44) and eqs. (6) -(7) show that, as soon as $\beta \neq 0$, baryons and DM have different dynamics. Neglecting radiation and any hydrodynamical effects, baryons move along geodesics. On the contrary, DM particles feel also non-gravitational forces and, as it happens in the presence of any non-gravitational interaction, do not follow geodesics. This is why baryon-DM segregation occurs.

Starting from eqs. (6) -(17), the evolution of linear fluctuations in cDE cosmologies was studied by [4]. Here we shall put ourselves into a different physical context, by lifting the restriction $\delta_{c, b} \ll 1$, but considering just cases when a full relativistic treatment is unessential. Then, dealing with scales well below horizon and with non-relativistic particles, the long-range force carried by the DE field $\phi$ can be described through corrections to newtonian gravity. They amount to assuming:

(i) DM particle masses to vary, so that

$$
M_{c}(\tau)=M_{c}\left(\tau_{i}\right) \exp \left[-C\left(\phi-\phi_{i}\right)\right]
$$

(ii) The gravitational constant between DM particles to become $G^{*}=\gamma G$.

Here

$$
C=\sqrt{16 \pi G / 3} \beta, \quad \gamma=1+4 \beta^{2} / 3
$$


A proof is given in Appendix A, following [12] and [6].

A newtonian treatment is suitable to study the growth of spherical top-hat fluctuations in the DM and baryon components and to show how their segregation occurs in the relevant physical cases. In fact, non-linearity starts well after matter-radiation decoupling and the top scales to be considered, galaxy cluster scales, lay well below the horizon.

The evolution of fluctuations in this regime was debated in paper I. Here we shall use its results to understand the shaping of mass functions. We therefore start from reviewing them and complementing them with new quantitative outputs.

\section{SPHERICAL TOP-HAT EVOLUTION}

Although in all cosmologies, apart sCDM, the system of equations ruling the spherical growth requires a numerical solution, in cDE models the numerical approach is far more essential. In fact, in all cases apart cDE, the only variable describing a top-hat fluctuation is its radius $R$. It is assumed to expand, initially, at the same rate of the scale factor $a$, obeying an equation similar to the scale factor in a closed model. The greater density inside the top hat slows then down the increase rates of $R$ in respect to $a$, so that the inner density $\rho(<R)$, although decreasing, becomes greater and greater than the average density $\rho$. Eventually, at a time $t_{t a}$, when the density contrast $\Delta=\rho(<R) / \rho$ attains a suitable value $\chi, R$ stops and starts decreasing. The equation it obeys would then cause $R$ to vanish within a finite time $t_{c}$. In sCDM cosmologies, $\chi=(3 \pi / 4)^{2}$ and $t_{c} / t_{t a}=2$. In other non-cDE cosmologies, $\chi$ and the ratio $t_{c} / t_{t a}$ take just slightly different values.

After $t_{t a}$, however, the very density inside $R$ is increasing, so that, in any realistic fluctuation, unless radiation disposes the heat produced by the $p d V$ work, virial equilibrium is attained and the collapse essentially stops when the fluctuation radius is $R_{v}$. In a sCDM cosmology this occurs for $R_{v} / R_{t a}=1 / 2$. Taking onto account the symultaneous growth of $a$, the virial density contrast is then $\Delta_{v}=32 \chi \simeq 180$. In other cosmologies the $R_{v} / R_{t a}$ ratio is slightly different from $1 / 2$ and $\Delta_{v}$ is mostly above 100 , but, in most cases, significantly below 180. A usual assumption is that the time when the system is stabilized into a virialized configuration coincides with $t_{c}$. Let us also remind that while, in $\mathrm{sCDM}, \Delta_{v}$ is independent from $t_{c}$, this is no longer true in all models where the density ratio between DE and material components depends on time. 
Quantitative results for the spherically symmetric growth in $\Lambda$ CDM models were given by 13]; their extension to models with uncoupled dDE is due to [14, 15]. In these cosmologies, the simple system of equations yielding spherical evolution requires a numerical solution.

Let us now consider the same problem in the cDE case. This problem had been considered in [16], but its approximated treatment did not allow the authors to focus the main physical effects. Following [1], let us outline, first of all, that linear fluctuations in baryons and DM are already different [12] and have a ratio

$$
\frac{\delta_{b}}{\delta_{c}} \simeq \frac{3 \Omega_{c}}{3 \gamma \Omega_{c}+4 \beta X \mu} .
$$

Here $X$ is given in eq. (8),$\gamma$ is given in eq. (10); $; \mu=\left(\dot{\delta}_{c, b} / \delta_{c, b}\right) / \mathcal{H}$. When considering a spherical top-hat fluctuation, whose initial radius $R_{T H, i}=R_{b}=R_{c}$, eq. (11) sets the initial ratio between DM and baryon fluctuation amplitudes.

Initial conditions are set so that both $R_{b}$ and $R_{c}$ initially grow as the scale factor. However, because of the different interaction strength, as soon as non-linear effects become significant, $R_{b}$ starts exceeding $R_{c}$. Hence, a part of the baryons initially within $R_{T H, i}$ leak out from $R_{c}$, so that DM does not feel the gravity of all baryons, while baryons above $R_{c}$ feel the gravity also of initially unperturbed DM layers. As a consequence, above $R_{c}$, the baryon component deviates from a top-hat geometry, while a secondary perturbation in DM arises also above $R_{c}$ itself.

After reaching maximum expansion, contraction will also start at different times for different components and layers. Then, inner layers will approach virialization before outer layers, whose later fall-out shall however perturb their virial equilibrium. This already outlines that the onset of virial equilibrium is a complex process.

Furthermore, when the external baryon layers fall-out onto the virialized core, richer of DM, they are accompanied by DM materials originally outside the top-hat, perturbed by baryon over-expansion.

The time when the greatest amount of materials, originally belonging to the fluctuation, are in virial equilibrium occurs when the DM top-hat has virialized, together with the baryon fraction still below $R_{c}$. A large deal of baryonic materials are then still falling out. But, when they will accrete onto the DM-richer core, they will not be alone, carrying with them originally alien materials. There will be no discontinuity in the fall-out process when all original baryons are back. The infall of outer materials just attains then a steady rate. 
In order to follow the dynamical evolution of a systems where each layer feels a different and substance-dependent force, a set of concentric shells, granting a sufficient radial resolution, needs to be considered. In the next section we shall provide the equations of motion for Lagrangian shell radii and review the whole expansion and recontraction dynamics.

\section{TIME EVOLUTION OF CONCENTRIC SHELLS}

\section{The spherical growth}

Dynamical equations can be written by using comoving radii $b_{n}=R_{b}^{n} / a\left(c_{n}=R_{c}^{n} / a\right)$ for the $n$-th baryon (DM) shell. Let $M_{b, c}^{n}$ be the masses of the $n$-th layer, $\bar{M}_{b, c}^{n}$ be the masses in the same layer in the absence of perturbations, and $\delta M_{b, c}^{n}=M_{b, c}^{n}-\bar{M}_{b, c}^{n}$ be the mass excesses for the $n$-th layer. Baryon shells keep a constant mass in the whole process, while

$$
M_{c}^{n}(\tau)=M_{c}^{n}\left(\tau_{i n}\right) \exp \left\{-C\left[\phi(\tau)-\phi\left(\tau_{i n}\right)\right]\right\}
$$

according to eq. (91). Similarly, let $\Delta M_{b, c}(<r)=M_{b, c}(<r)-\bar{M}_{b . c}(<r)$ be the mass excess within the physical radius $R=a r$ and let then be

$$
\Delta M_{b}^{n}=\Delta M_{b}\left(<b_{n}\right)+\Delta M_{c}\left(<b_{n}\right), \quad \Delta M_{c}^{n}=\Delta M_{b}\left(<c_{n}\right)+\gamma \Delta M_{c}\left(<c_{n}\right),
$$

including a correction aimed to take into account, besides of the mass variation (eq. 12), also that $G^{*} \neq G$ in DM-DM interactions.

A systematic discrepancy will arise between $b_{n}$ and $c_{n}$ and will require taking into account only the fraction of the last shell below the radius considered. In paper I it is shown that the shell dynamics can then be described through the equations

$$
\ddot{c}_{n}=-\left(\frac{\dot{a}}{a}-C \dot{\phi}\right) \dot{c}_{n}-G \frac{\Delta M_{c}^{n}}{a c_{n}^{2}}, \quad \ddot{b}_{n}=-\frac{\dot{a}}{a} \dot{b}_{n}-G \frac{\Delta M_{b}^{n}}{a b_{n}^{2}},
$$

to be integrated together with the first of eqs. (44) and the Friedman equation.

Until $b_{n} \sim c_{n}$, it is $\Delta M_{b}^{n}<\Delta M_{c}^{n}$, for any $\beta>0$. Hence, the gravitational push felt by DM layers is stronger. The extra term $C \dot{\phi} \dot{c}_{n}$ adds to this push. In fact, the comoving variable $c_{n}$ has however a negative derivative (see the lower panels in Figure 1). As a consequence, $c_{n}$ decreases more rapidly than $b_{n}$, so that the $n$-th baryon radius may gradually exceed the $(n+1)$-th DM shell, etc. . As a consequence, the sign of $\Delta M_{b}^{n}-\Delta M_{c}^{n}$ can even invert and 

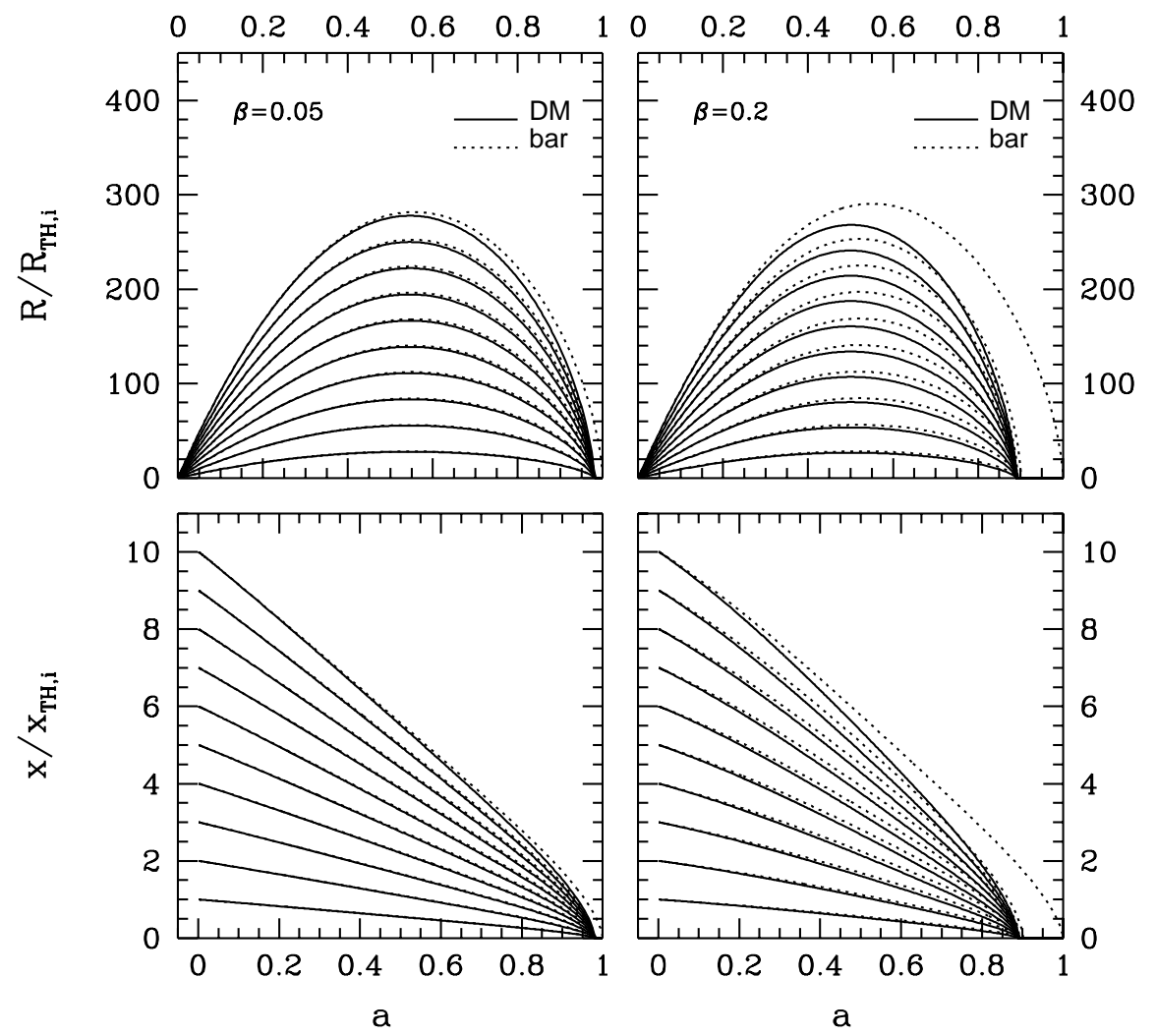

FIG. 1: Evolution of a sample of baryon and DM layer radii, extending up to the top-hat radius; upper (lower) panels describe physical (comoving) radii. In all plots the leaking out of the upper baryon layer is clearly visible.

one can evaluate for which value of $\phi-\phi_{i}$ this occurs. Once $\phi(\tau)$ is known, also the time when this occurs can be found. Until then, however, DM fluctuations expand more slowly and mostly reach their turn-around point earlier, while baryons gradually leak out from the fluctuation bulk.

The whole behavior is visible in the Figure 1 for samples of $R_{c, b}$ and $c-b$ (the ordinate label $\mathrm{x}$ stands for either $c$ or $b$ ). The greatest radii shown are the baryon top-hat radii. Solid (dotted) lines yield the $a$ dependence for DM (baryon) shell radii. The actual number of radii $R_{b, c}^{n}$ used in the equations depend on the precision wanted; radii do not need to be equi-spaced. To our aims (a $0.1 \%$ precision), $\sim 1000$ radii were sufficient. If more precision is needed, it can be easily achieved at the expenses of using a greater computer time. With a single processor 1.5 Gflops $\mathrm{PC}$, our optimized program takes $\sim 60$ minutes to run a single case, with $\sim 1000$ radii. 


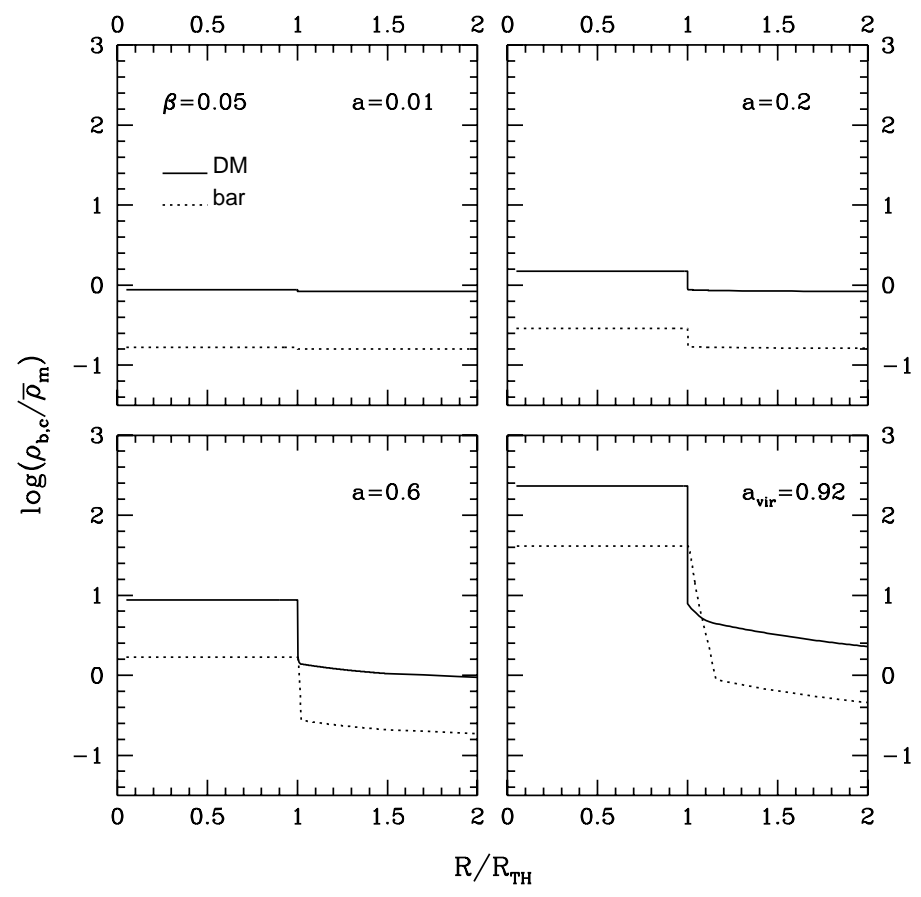

FIG. 2: Density profiles at different $a$ values for a cDE model with $\beta=0.05$. Solid (dotted) lines refer to DM (baryons). Notice the progressive deformation of the baryon profile (dotted lines) in respect of the DM profile (solid line).

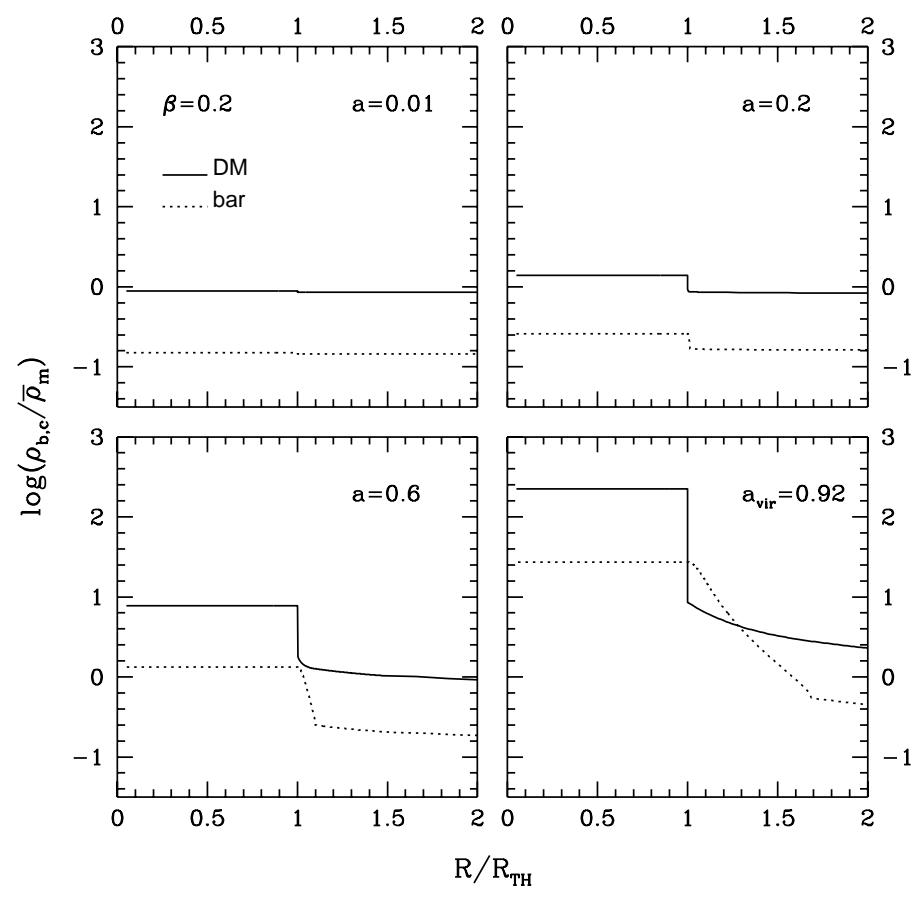

FIG. 3: Density profiles at different $a$ values for $\beta=0.2$ model. The deformation of the baryon profile (dotted lines) in respect of the DM profile (solid line) is stronger than in the previous Figure. 
The full collapse shown in Figure 1 is not expected in any physical case, as the $R_{n}$ decrease stops when virialization is attained. Figs. 2, 3] therefore assume that the spherical growth stops when all DM originally in the top-hat, and the baryons kept inside it, virialize. This figure describes the gradual deformation of the top-hat profile. Already at $a=0.2$, well before the turn-around, the slope of the top-hat boundary, for baryons, is no longer vertical. At $a=0.6$ (approximately turn-around), not only the baryon boundary is bent, but a similar effect is visible also for DM. The effect is even more pronounced at $a_{v i r} \simeq 0.92$. Figures similar to the upper plots in Fig. 1 and to Figs. 2, 3. were already shown in paper I, although for different model parameters.

The increased density of DM shells outside the top-hat is relevant, here, because it fastens the recollapse of outer baryons. In turn, the enhanced baryon density, outside from DM top-hat, modifies the dynamics of external DM layers, as well.

The most significant physical effect, however, is the outflow of baryon layers from the DM top-hat. The outflown baryon fraction increases with $a$. For $a \sim 0.92$, i.e. when DM and inner baryons have attained their virialization radius, the fraction of baryons which have leaked out from the fluctuation is so large as $\sim 10 \%$, even for $\beta=0.05$; it reaches $\sim 40 \%$ for $\beta=0.2$. These values increase by an additional $10 \%$ in the RP case.

\section{Virialization}

In Figures 2 and ?? we assumed the present time to coincide with virialization for all DM inside the top-hat fluctuation and all baryons kept inside it, excluding therefore a large deal of baryons, either 10 or $40 \%$, initially inside the top-hat, but leaking out during the fluctuation growth.

It must also be outlined that much care ought to be taken to define the virialization condition, for materials within any radius $R$, reading

$$
2 T(R)=R d U(R) / d R
$$

by summing up DM and baryon kinetic energies

$$
T_{c}(R)=2 \pi \int_{0}^{R} d r r^{2} \rho_{c}(r) \dot{r}^{2}, \quad T_{b}(R)=2 \pi \int_{0}^{R} d r r^{2} \rho_{b}(r) \dot{r}^{2}
$$

and taking into account that, during the whole fluctuation evolution, potential energies include three terms, due to self-interaction, mutual interaction, interaction with the DE 
field. More in detail, for DM and baryons, we have

$$
\begin{aligned}
& U_{c}(R)=U_{c c}(R)+U_{c b}(R)+U_{c, D E}(R)=4 \pi \int_{0}^{R} d r r^{2} \rho_{c}(r)\left[\bar{\Psi}_{c}(r)+\Psi_{b}(r)+\Psi_{D E}(r)\right] \\
& U_{b}(R)=U_{b b}(R)+U_{b c}(R)+U_{b, D E}(R)=4 \pi \int_{0}^{R} d r r^{2} \rho_{b}(r)\left[\Psi_{b}(r)+\Psi_{c}(r)+\Psi_{D E}(r)\right]
\end{aligned}
$$

respectively. While

$$
\Psi_{b}(r)=-\frac{4 \pi}{3} G \rho_{b}(r) r^{2}, \quad \Psi_{D E}(r)=-\frac{4 \pi}{3} G \rho_{D E}(r) r^{2}
$$

in both expressions, a subtle difference exists for $\Psi_{c}$. In $U_{b}$ we have simply

$$
\Psi_{c}(r)=-\frac{4 \pi}{3} G \rho_{c}(r) r^{2}
$$

as for the other components, but this expression is different in $U_{c}$, where it reads

$$
\bar{\Psi}_{c}(r)=-\frac{4 \pi}{3}\left\{\gamma G\left[\rho_{c}-\bar{\rho}_{c}(r)\right]+G \bar{\rho}_{c}\right\} r^{2},
$$

$\bar{\rho}_{c}$ being the background DM density. The different dynamical effect of background and DM fluctuation arises from the different ways how its interaction with DE is treated. Energy exchanges between DM and DE, for the background, are accounted for by the r.h.s. terms in eqs. (41). In this case no newtonian approximation was possible and was made. For DM fluctuation, instead, the effects of DM-DE exchanges are described by a correction to the gravitational constant $G$, becoming $G^{*}=\gamma G$, which adds to the dependence of DM density on $\phi$. This ought to be taken into account in the fluctuation evolution, as is done in eq. (21).

The above expressions hold for any $R$. However, if $R$ coincides with DM top-hat (or is smaller than it), the densities $\rho_{b, c, D E}$ do not depend on $r$. Densities depend on $r$ if one aims to take somehow into account the shells where baryons, initially belonging to the top-hat, have flown. In the former case, the integrals (17), (18) and (16) can be easily performed and yield

$$
\begin{gathered}
U_{c}(R)=(3 / 5) G\left[M_{c}\left(M_{b}+\bar{M}_{c}\right)+\gamma M_{c} \Delta M_{b}\right] / R-(4 \pi / 5) M_{c} \rho_{D E} R_{2} \\
U_{b}(R)=-(3 / 5) G\left[M_{b}\left(M_{b}+M_{c}\right)\right] / R-(4 \pi / 5) M_{b} \rho_{D E} R^{2} \\
T_{c}(R)=(3 / 10) M_{c} \dot{R}^{2}
\end{gathered}
$$


where the relation $\dot{r} / r=\dot{R} / R$ is used to calculate $T_{c}(R)$. Note that this relation is not valid for $T_{b}(R)$ because different baryon layers have different growth rates. Kinetic energy for baryons is then obtained by

$$
T_{b}(R)=\sum_{n} T_{b}^{n}=\sum_{n} \frac{1}{2} M_{b}^{n}\left(\dot{R}_{b}^{n}\right)^{2}
$$

Here the sum is extended on all $R_{b}^{n}<R$.

If integrals are extended above the DM top-hat, recourse to numerical computations is needed.

\section{MASS FUNCTIONS IN cDE THEORIES}

The expected physical behavior of a top-hat fluctuation, including non-linear features, can be compared with the evolution of that fluctuation if we assume that linear equation however hold, indipendently of the actual amplitude the fluctuation has reached. Actual gravitation prescribes that fluctuations approaching unity abandon the linear regime, slow down their expansion rate, reach maximum expansion, turn-around and recontract, finally recollapsing to nil. While this occur, we can formally assume that linear equation still hold and seek the value $\delta_{r c}$ that linear fluctuations would have at the time $\tau_{r c}$ when, according to actual gravitation, they have recollapsed.

Let us also remind that the linear evolution does not affect fluctuation amplitude distributions. Accordingly, if fluctuation amplitudes are distributed in a Gaussian way, this does not change in the linear regime. Therefore, at the time $\tau_{r c}$, we can integrate on the distribution of fluctuation amplitudes for a given scale, taking those $>\delta_{r c}$, so finding the probability that an object has formed and virialized over such scale.

As already outlined in the previous section, full recollapse is not expected to occur. The usual assumption is however that the time running between the achievement of the virialization prescription and the time formally required for full recollapse is taken by the fluctuation to achieve a relaxed virial equilibrium configuration. This is the basic pattern

of the PS-ST approach, that we aim now to apply to cDE cosmologies. In the presence of DM-DE coupling, however, a novel feature must be considered: starting from the initial amplitudes set by the linear theory, DM and baryon fluctuations require different times to reach full recollapse. Accordingly, if we require a baryon fluctuation to reach full recollapse 


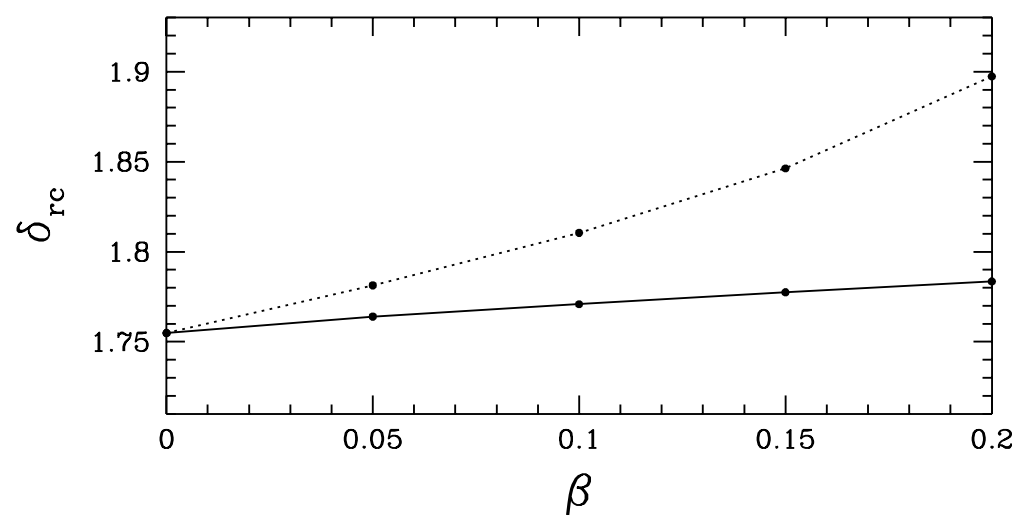

FIG. 4: $\beta$ dependence of $\delta_{c, r c}^{(c)}$ (dotted line) and $\delta_{c, r c}^{(b)}$, at $z=0$ (solid line).

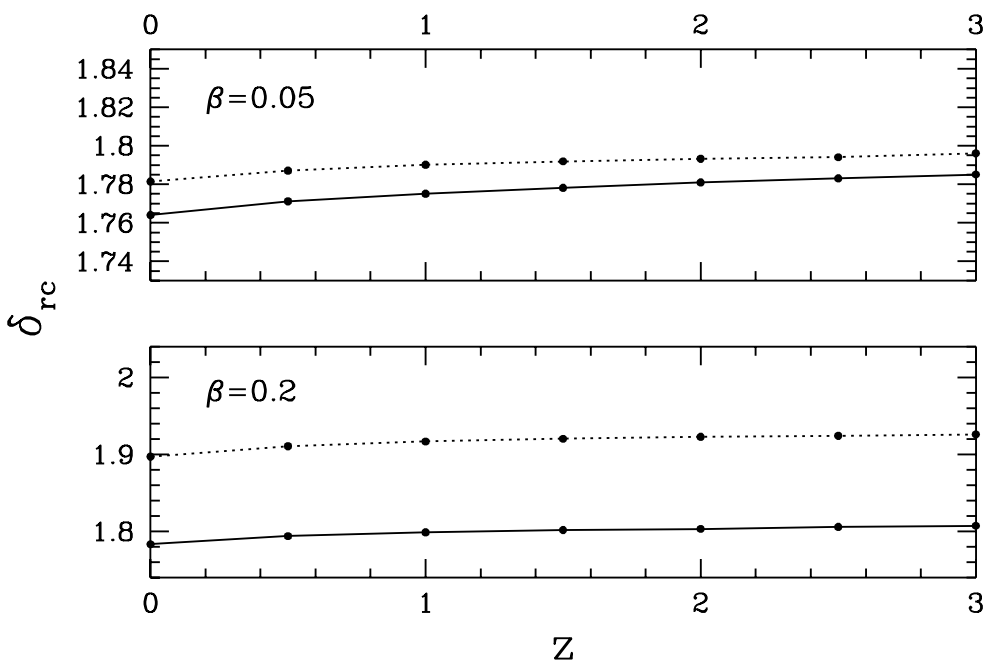

FIG. 5: Redshift dependence of $\delta_{c, r c}^{(c)}$ (dotted line) and $\delta_{c, r c}^{(b)}$ (solid line).

at the present time $\tau_{o}$ (or at any time $\tau$ ), the initial linear $\delta_{b}$ and $\delta_{c}$ must have been greater than those required to allow full recollapse at the present time $\tau_{o}$ (or at any time $\tau$ ) for a DM fluctuation.

Let us also remind that the linear theory does not prescribe equal linear amplitudes for DM and baryons, but that their ratio is given by eq. (11). Therefore, it becomes also important to outline that we chose to refer to DM fluctuation amplitudes in the linear regime. Accordingly, let $\delta_{c, r c}^{(c)}$ and $\delta_{c, r c}^{(b)}$ be both DM fluctuation amplitudes, defined so that, if the linear theory yields the former (latter) value at a time $\tau$, the corresponding DM (baryon) fluctuation has fully recollapsed at $\tau$. 

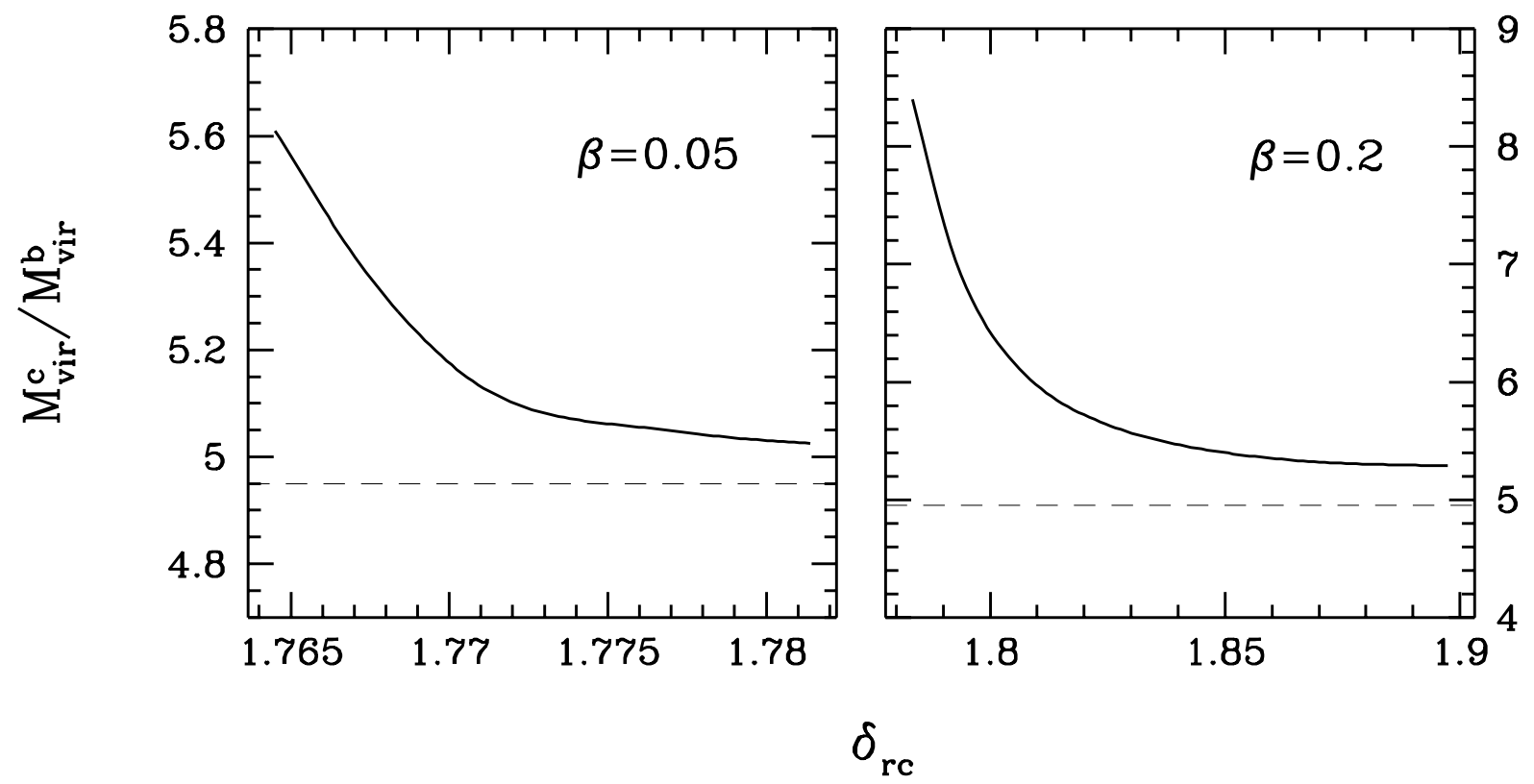

FIG. 6: Ratio between DM and baryon mass in virialized halos, against the value used for $\delta_{c, r c}$ (DM masses are rescaled to the value they reach at $\tau_{o}$. The dashed line is the background $\Omega_{c} / \Omega_{b}$ ratio.

In Figure 4 we plot the $\beta$ dependence of $\delta_{c, r c}^{(c)}$ and $\delta_{c, r c}^{(b)}$, at $z=0$. They start from equal values for $\beta=0$ and gradually split as $\beta$ increases. In Figure 5 we plot the $z$ dependence of $\delta_{c, r c}^{(c)}$ and $\delta_{c, r c}^{(b)}$ for $\beta=0.20$ and 0.05 . These values were computed by starting at $z=1000$, taking into account also the radiative component, and using the full set of equations.

Following the PS approach, the differential mass function then reads

$$
\begin{aligned}
\psi(M) & =2 \frac{\rho}{M} \int_{\delta_{c, r c}}^{\infty} d \delta_{M} \frac{d \sigma_{M}}{d M} \frac{d}{d \sigma_{M}}\left\{\frac{1}{\sqrt{2 \pi} \sigma_{M}} e^{-\delta_{M}^{2} / 2 \sigma_{M}^{2}}\right\} \\
& =\sqrt{\frac{2}{\pi}} \frac{\rho}{M} \int_{\delta_{c, r c} / \sigma_{M}}^{\infty} d \nu_{M} \frac{d \nu_{M}}{d M} \nu_{M} e^{-\nu_{M}^{2} / 2} .
\end{aligned}
$$

Here $\nu_{M}=\delta_{M} / \sigma_{M}$ and $\delta_{c, r c}$ can be either $\delta_{c, r c}^{(c)}$ or $\delta_{c, r c}^{(b)}$ (or any intermediate value) according to Fig. 4 and 5 a choice shall be based on the observable to be fitted.

The ST expression is obtainable from eq. (26) through the replacement

$$
\begin{gathered}
\nu_{M} \exp \left(-\nu_{M}^{2} / 2\right) \rightarrow \mathcal{N}^{\prime} \nu^{\prime}{ }_{M}\left(1+\nu_{M}^{\prime-3 / 5}\right) \exp \left(-\nu^{\prime 2}{ }_{M} / 2\right), \\
\text { with } \quad \mathcal{N}^{\prime}=0.322, \quad \nu^{\prime 2}{ }_{M}=0.707 \nu_{M}^{2}
\end{gathered}
$$


meant to take into account the effects of non-sphericity in the halo growth.

In the absence of coupling and baryon-DM segregation, the mass $M$ in the PS and ST expressions (26)-(27) is the mass originally in the top-hat, which will then be comprised within a virial radius $R_{v}$, such that $M /(4 \pi / 3) \rho R_{v}^{3}=\Delta_{v}$.

In the presence of coupling and segregation the situation is more complex. However, indipendently of the value taken for $\delta_{c, c r}$, the resulting virialized system will be baryon depleted. Different possible $\delta_{c, c r}$ 's will correspond to different depletions, but the final system shall however contain a smaller fraction of baryons, in respect the background $\Omega_{b} / \Omega_{c}$ ratio.

It is important to distinguish between two effects: (i) DM mass variation. (ii) The dynamics of gravitational growth. Let $M_{i}$ and $M_{v i r}$ be the masses, at the initial time and at virialization, rescaled to the values they will have at $\tau_{o}$, so that the (i) effect is isolated. Then, while

$$
M_{i}=M_{i}^{c}+M_{i}^{b}=\left(\Omega_{c} / \Omega_{m}\right) M_{i}+\left(\Omega_{b} / \Omega_{m}\right) M_{i},
$$

so that $M_{i}^{c} / M_{i}^{b}=\Omega_{c} / \Omega_{b}$, in the decomposition

$$
M_{v i r}=M_{v i r}^{c}+M_{v i r}^{b}
$$

it will however be $M_{v i r}^{c} / M_{v i r}^{b}>\Omega_{c} / \Omega_{b}$.

Let us now duly take into account also the (i) effect and consider the case when the mass function is set by $\delta_{c, r c}^{(c)}$. Then, while $M_{v i r}^{c} / M_{i}^{c}=\exp \left[-C\left(\phi_{v i r}-\phi_{i}\right)\right]$, it will obviously be $M_{v i r}^{b}<M_{i}^{b}$ : several baryon layers, initially belonging to the fluctuation, have not yet recollapsed or virialized.

Let us then consider the mass function set by $\delta_{c, r c}^{(b)}$. In this case it is $M_{v i r}^{b}=M_{i}^{b}$, but it will be $M_{v i r}^{c} / M_{i}^{c}>\exp \left[-C\left(\phi_{v i r}-\phi_{i}\right)\right]$. The extra DM mass is due to those layers, initially external to the fluctuation, first compressed and then conveyed inside the virialization radius, together with the baryons previously outflown from the DM bulk.

For any $\delta_{c, r c}$ in the $\delta_{c, r c}^{(c)}-\delta_{c, r c}^{(b)}$ interval, some baryon layers will still be out and some extra DM will have been conveyed inside the virial radius by the fall out of outflown baryons. Hence, it will however be $M_{v i r}^{c} \exp \left[-C\left(\phi_{o}-\phi_{v i r}\right] / M_{v i r}^{b}>\Omega_{c} / \Omega_{b}\right.$.

In Figure [6] we plot this ratio, as a function of $\delta_{c, r c}$, in the $\delta_{c, r c}^{(c)}-\delta_{c, r c}^{(b)}$ interval. The plot shows that, after a fast decrease, the ratio tends to a steady value, however exceeding the background ratio. The curves shown in this plot depend on the assumed (top-hat) shape for the primeval fluctuation, but similar curves would hold for any initial shape. 
A prediction of cDE theories, therefore, is that $\Omega_{c} / \Omega_{b}$, measured in any virialized structure, exceeds the background ratio. The excess is greater for structures where only DM has virialized. They might be characterized by an apparent disorder in the baryon component, still unsettled in virial equilibrium while, e.g., a lensing analysis would show that they are safely bound systems.

We shall now plot mass functions obtained using either $\delta_{c, r c}^{(c)}$ or $\delta_{c, r c}^{(b)}$. We expect actual measures to yield a value comprised in this interval and, however, closer to the $\delta_{c, r c}^{(c)}$ curve when baryon stripping is stronger. In Figure [7 we plot the integral mass functions $n(>M)=\int_{M}^{\infty} d M^{\prime} \psi\left(M^{\prime}\right)$ obtained through ST expressions (26)-(27). Let us remind that large differences between models were never found in mass functions at $z=0$, because of DE nature. The upper panel of each figure shows the mass function in the usual fashion, as often plotted to fit data or simulations. In the lower panel we plot the ratio between expected halo numbers for each model and $\Lambda$ CDM. This confirms the small shifts between $\Lambda \mathrm{CDM}$ and $\mathrm{dDE}$ cosmologies, yielding just a slight excess, $\sim 10 \%$, on the very large cluster scale, where observed clusters are a few units.

Discrepancies can be more relevant between $\Lambda$ CDM and cDE, whose effective mass function should however lie inside the dashed areas, limited by the function obtained by integrating from $\delta_{c, r c}^{(c)}$ or $\delta_{c, r c}^{(b)}$. The plots show a shortage of larger clusters. For $\beta=0.20$, they are half of $\Lambda \mathrm{CDM}$ at $\sim 3 \cdot 10^{14} h^{-1} M_{\odot}$ and really just a few above some $10^{15} h^{-1} M_{\odot}$. Any realistic mass function, laying in the dashed area, can be falsified by samples just slightly richer than those now available.

For $\beta=0.05$ the shift is smaller, hardly reaching $20 \%$, still in the direction opposite to dDE. Here we meet what appears to be a widespread feature of cDE models: the discrepancy of $\mathrm{dDE}$ from $\Lambda \mathrm{CDM}$ is partially or totally erased even by a fairly small DM-DE coupling, and many cDE predictions lay on the opposite side of $\Lambda \mathrm{CDM}$, in respect to dDE. If a $\Lambda \mathrm{CDM}$ model is then used to fit galaxy or cluster data arising in a cDE cosmology, we expect cluster data to yield a best-fit $\sigma_{8}$ value smaller than the one obtained by fitting galaxy data.

The integral mass functions obtained through the original PS expression are shown in Fig. 8. This figure shows just slight quantitative shifts. In the cases illustrated by the next figures, results from PS expression will be therefore omitted.

Figure 9 compares RP results with SUGRA, at $z=0$. Quantitative differences exist, when the self-interaction potential is changed, but most physical aspects are the same. 

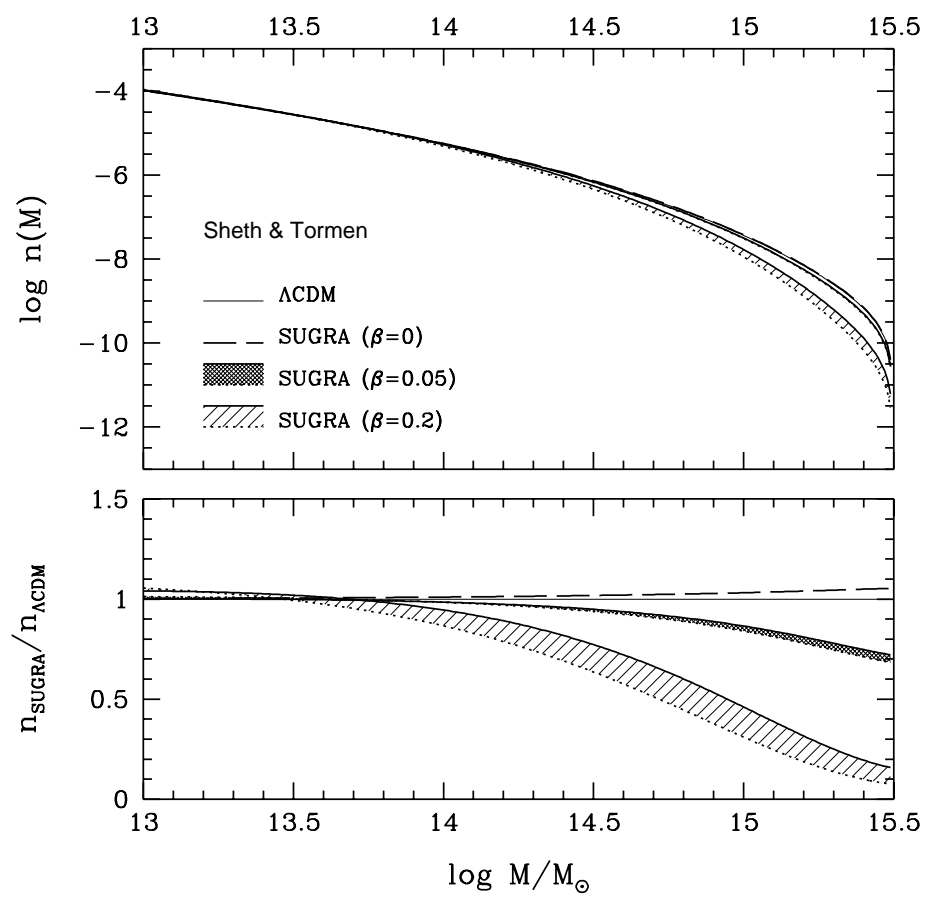

FIG. 7: Cluster per $\left(\operatorname{Mpc} h^{-1}\right)^{3}$, above mass $M$ at $z=0$, obtained from ST expression. Four models are considered, with equal $\Omega$ 's and $h$ : $\Lambda$ CDM, uncoupled SUGRA with $\Lambda=100 \mathrm{MeV}$, and two $\mathrm{cDE}$ with different $\beta$ 's. The dashed areas are limited by the mass functions worked out for $\delta_{c, r c}^{(c)}$ or $\delta_{c, r c}^{(b)}$ in cDE models (see text).
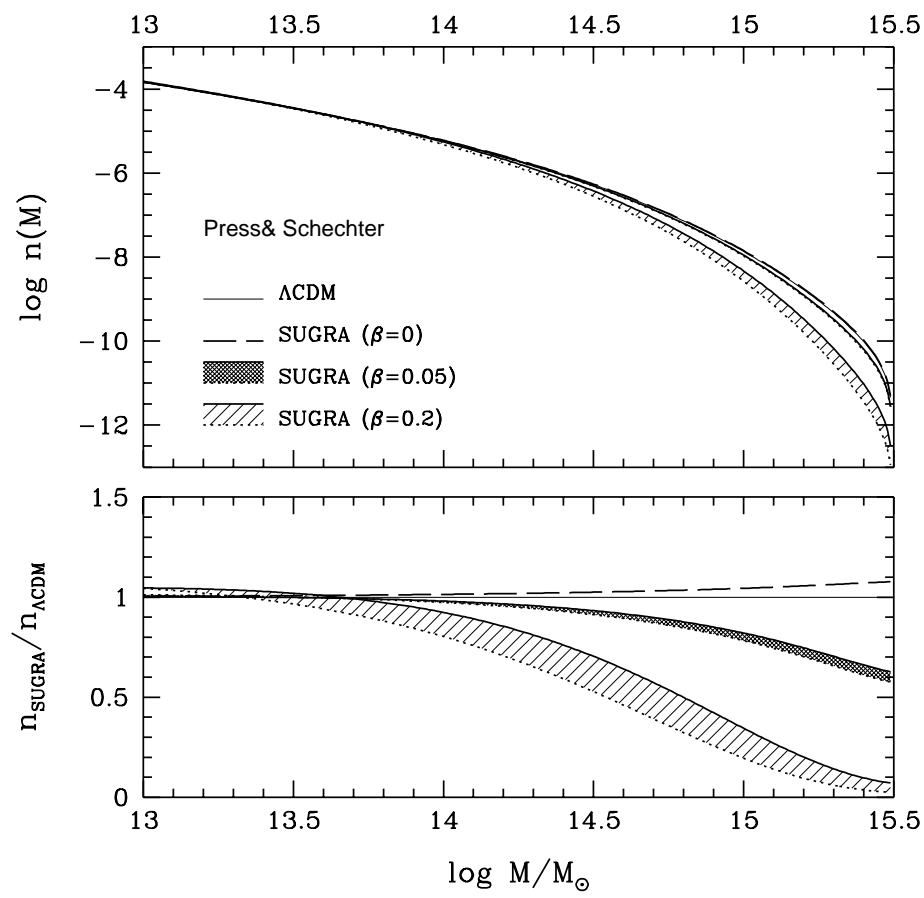

FIG. 8: The same as Fig. [7 using the original PS expression. This figure shows just slight quantitative shifts. 


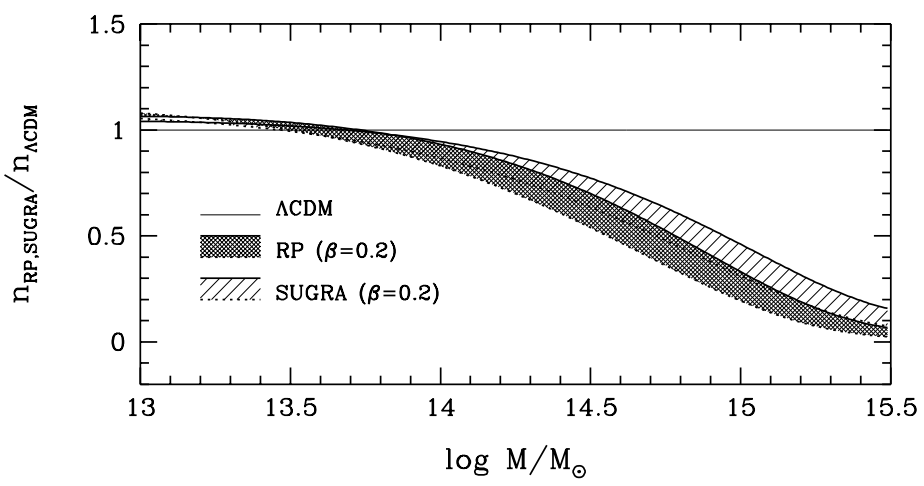

FIG. 9: A comparison of SUGRA and RP results at $z=0$ using the ST expression. The lower panel of Fig. 7 is reproduced, neglecting dDE and $\beta=0.05$ cases and adding RP results (heavy dashed).
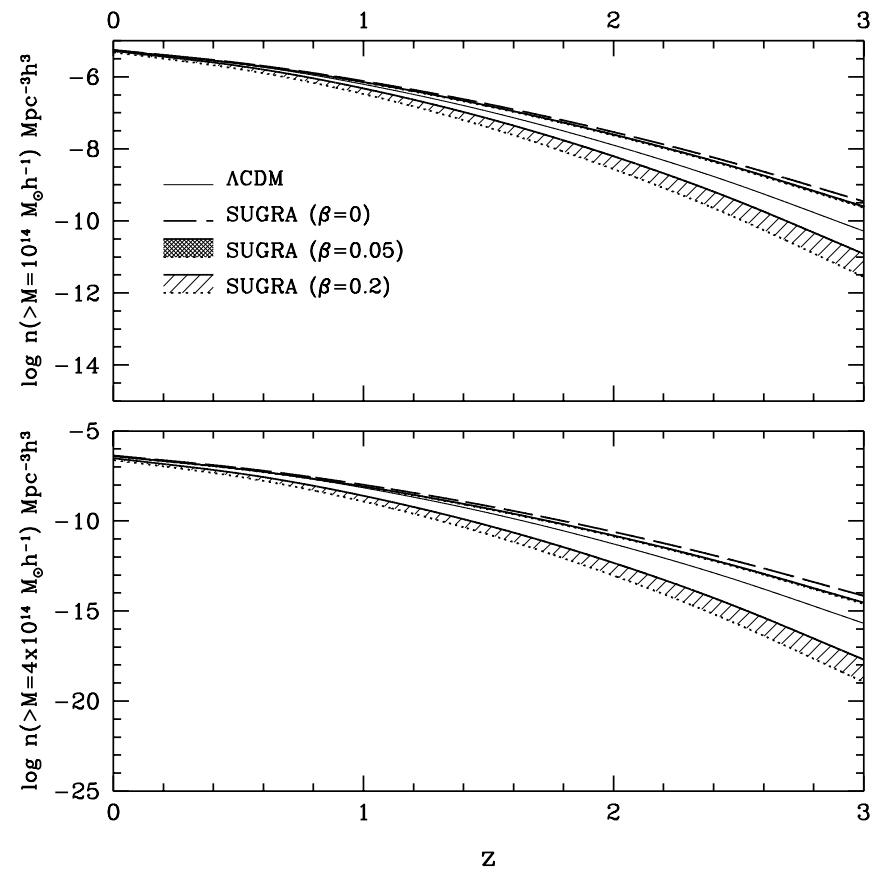

FIG. 10: Cluster per $\left(\operatorname{Mpc} h^{-1}\right)^{3}$, above mass $M$ at different $z$ 's, in a fixed comoving volume, obtained from ST expression. Thick solid and dotted lines refer to cDE models. The dashed (thinner solid) line refers to $\mathrm{dDE}(\Lambda \mathrm{CDM})$. 


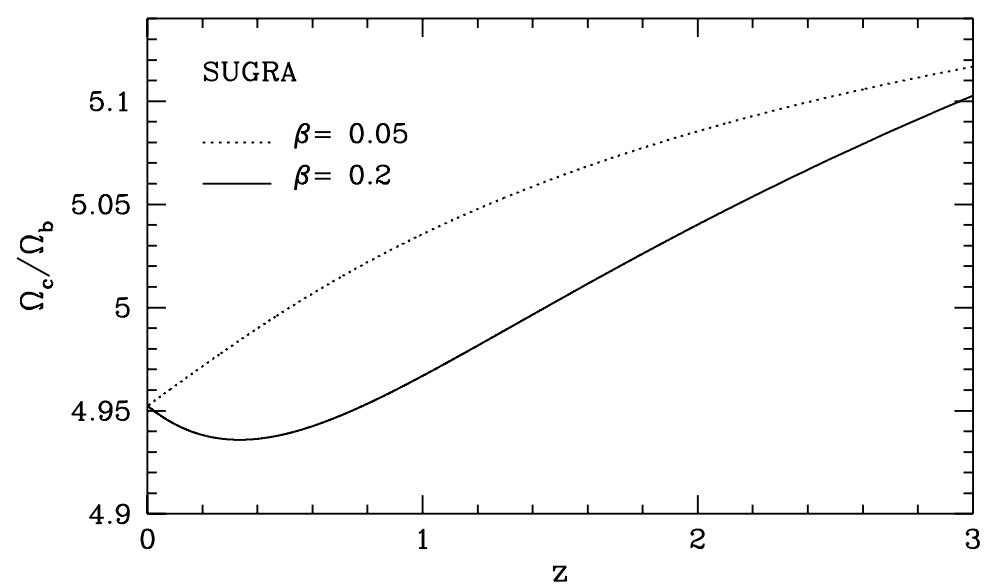

FIG. 11: Evolution of the DM/baryon background ratio, due to the dynamics of the $\phi$ field.

In Figure 10 the redshift dependence of the expected cluster numbers in a comoving volume is plotted against the redshift $z$, for $M=10^{14}$ and $4 \cdot 10^{14} h^{-1} M_{\odot}$. As usual, the mass considered is the total cluster mass. As already widely outlined, the DM/baryon ratio in these masses however exceeds the background $\Omega_{b} / \Omega_{c}$ ratio; the spread of the function corresponds to the spread of possible baryon/DM ratios. In top of that, however, one must also remind that the very background $\Omega_{c} / \Omega_{b}$ ratio varies with redshift, because of the evolution of the $\phi$ field. Hence, clusters observed at high $z$, in average, shall be however baryon poorer than present time clusters. The $z$ dependence of the background $\Omega_{c} / \Omega_{b}$ ratio, for the model considered in this work, is plotted in Figure 11.

Plots similar to Figure 10 are often used to assert the possibility to discriminate between models and this plot is however significant to compare cDE with former results for other cosmologies. According to [17], however, the discriminatory capacity of this observable can only be tested by plotting cluster numbers per solid angle and redshift interval, which also includes geometrical effects, often partially erasing dynamical effects.

In the upper panels of Figures 12 and 13 cluster numbers per solid angle and redshift interval are plotted. In the lower panels we plot the ratios between each SUGRA model and $\Lambda \mathrm{CDM}$ mass functions. We consider again the mass scales $10^{14}$ and $4 \cdot 10^{14} h^{-1} M_{\odot}$; for the latter mass we provide a magnified box to follow the expected low-z behavior. Notice, in particular, that the high-z behaviors for $\beta=0.05$ or 0.2 , for these mass scales, lay on the opposite sides of $\Lambda$ CDM. The box in Fig. 13] show how we pass from numbers smaller than 

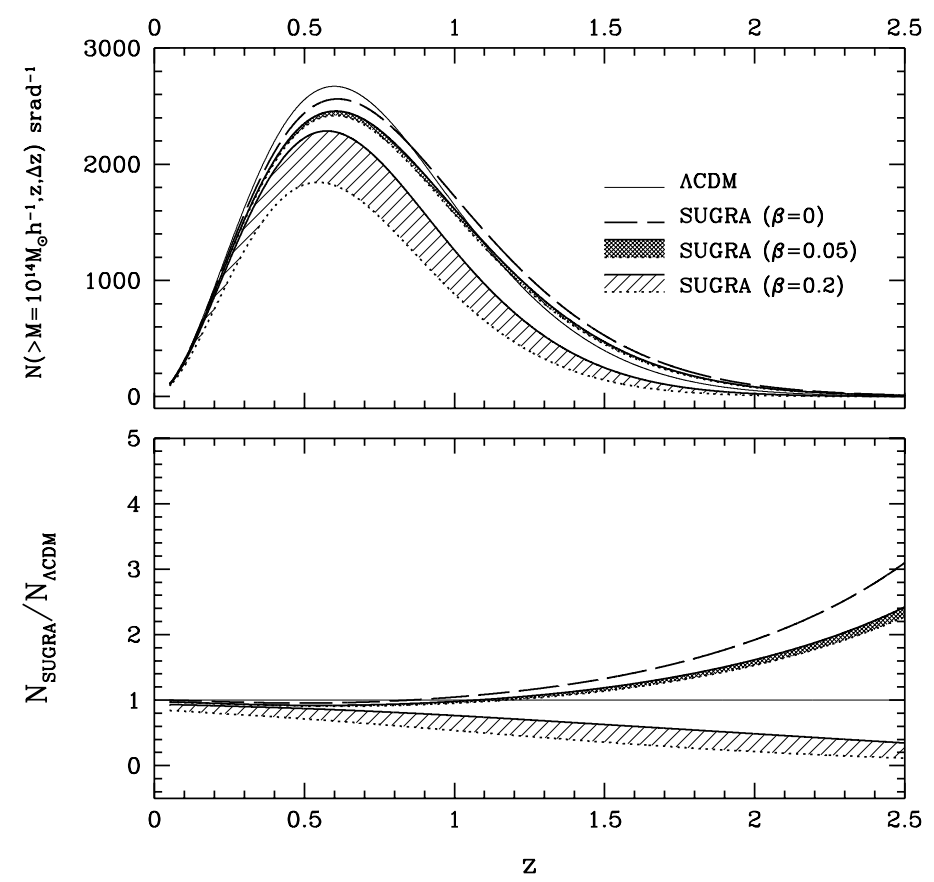

FIG. 12: Number of clusters with $M>10^{14} h^{-1} M_{\odot}$ in a fixed solid angle and redshift interval. Lines as in Fig. 10.
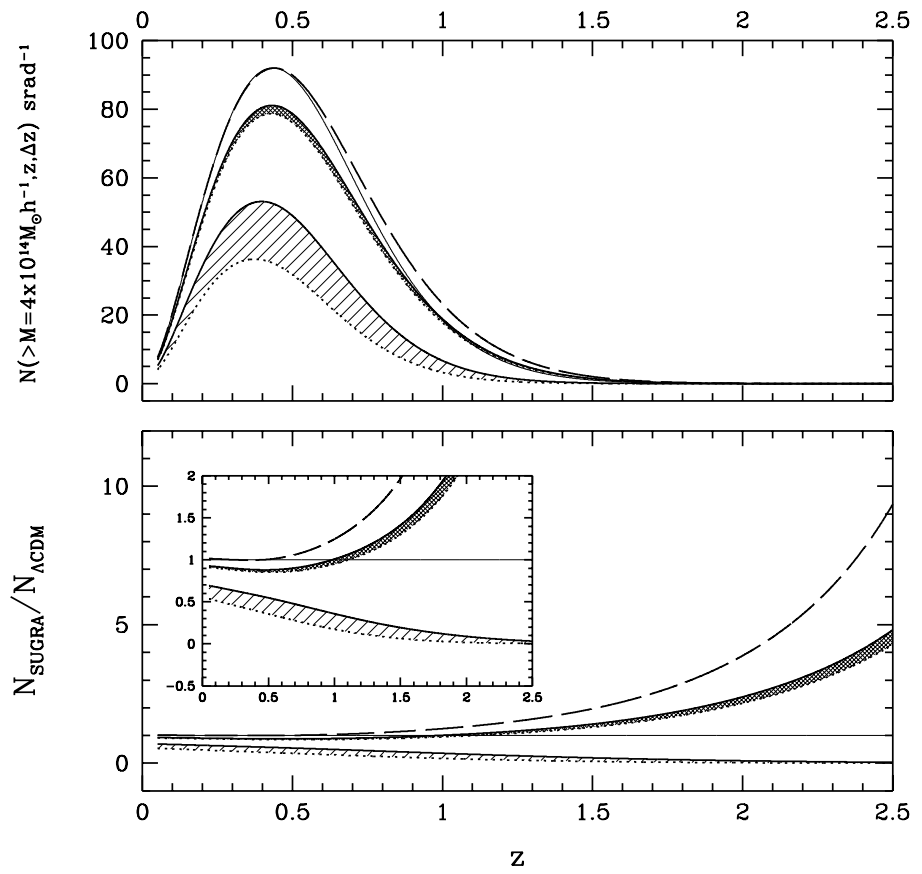

FIG. 13: Number of clusters above $4 \cdot 10^{14} h^{-1} M_{\odot}$. Lines and comments as for Fig. 12 , 

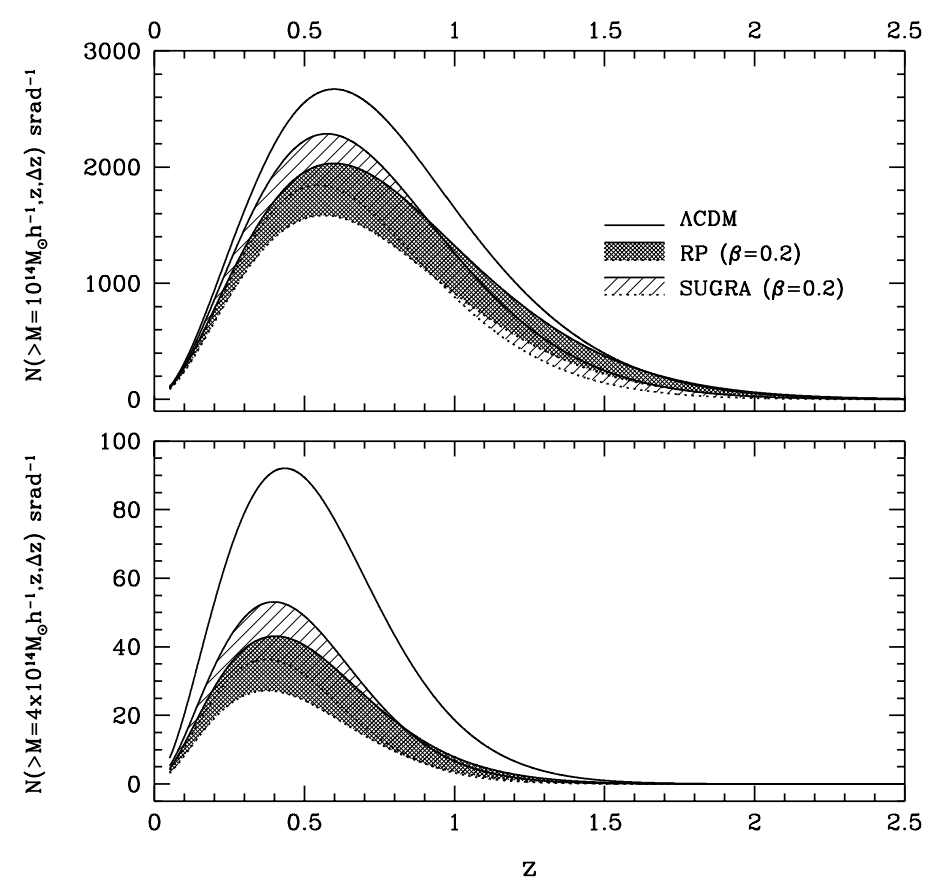

FIG. 14: A comparison between SUGRA and RP results. Here the top panels of Figs. 12 and 13 are reproduced, omitting the $\beta=0.05$ case and showing both SUGRA (dashed) and RP (heavy dashed) number intervals. The number distribution of $\Lambda \mathrm{CDM}$ is also given (solid line).

$\Lambda \mathrm{CDM}$ to greater numbers, for $\beta=0.05$, at a redshift $z \simeq 0.7$.

As is obvious, a better discrimination is attained for high masses or deep redshifts. However, according to Fig. [13, a sample including a few dozens clusters of mass $>4 \cdot 10^{14} h^{-1} M_{\odot}$ at $z>0.5$ would already bear a significant discriminatory power.

Altogether we see that, (i) when passing from $\Lambda$ CDM to uncoupled SUGRA, the high- $z$ cluster number is expected to be greater. (ii) When coupling is added, the cluster number excess is reduced and the $\Lambda \mathrm{CDM}$ behavior is reapproached. (iii) A coupling $\beta=0.05$ may still yield result on the upper side of $\Lambda \mathrm{CDM}$, while $\beta=0.2$ displaces the expected behavior well below $\Lambda$ CDM. The $\Lambda$ CDM behavior is approsimately met for $\beta=0.1$.

These behaviors arise, first of all, from the different evolution of $\rho_{D E}$ at high $z$. In $\Lambda$ CDM models, $\rho_{D E}$ simply keeps constant, while $\rho_{m} \propto(1+z)^{3}$, and therefore its relevance rapidly fades. In $\mathrm{dDE}$ models, instead, $\rho_{D E}$ increases with $z$, although at a smaller rate than $\rho_{m}$. To obtain the same amount of clusters at $z=0$ requires that they have existed since earlier.

When coupling is added, however, gravitation is boosted by the $\Phi$ field. In the newtonian language, this translates into a greater gravity constant $\left(G^{*}\right)$ and growing masses for DM 
particles. This speeds up cluster formation and, when $\beta$ increases, less clusters are needed at high $z$, to meet their present numbers.

Finally, in Figure 14 we provide a comparison between the PS-ST mass function obtainable for RP and SUGRA potentials. Only the case $\beta=0.2$ is considered. A RP potential slightly strengthens the effects already seen for SUGRA; as a matter of fact, however, in most cases we find just small quantitative shifts.

\section{CONCLUSIONS}

In this work we aimed at predicting the cluster mass function in cDE cosmologies, by using the solution of the equations ruling the spherical growth of top-hat fluctuations in ST (or PS) expressions. The effectiveness of ST expressions has been widely verified for SCDM, $\Lambda \mathrm{CDM}$ and 0CDM models. Also in simulations of models with dynamical (uncoupled) DE 19], ST expressions provide a fair fit of numerical outputs.

The main finding of this study is the significant baryon-DM segregation, which has multiple effects. If cluster numbers are measured from their gravitational effects, e.g., by using lensing data, a PS-ST approach yields simple predictions. When cluster numbers are measured through other observables, we predict a number range, inside which observations should lie. The actual amount of objects, inside these ranges, is determined by a number of effects, that a PS-ST approximation cannot describe.

An important example of such effects is the possible stripping of outer layers, in close encounters, which will mostly act on the baryon component. For a rather small coupling as $\beta=0.2$, up to $40 \%$ of the baryons belonging to the initial fluctuation could be stripped in this way and, even for the tiny coupling set by $\beta=0.05,10 \%$ of baryons could be easily stripped.

If cluster data are obtained from galaxy counts or hot gas features, they will exhibit the residual baryon amount. Indipendently of the baryon loss, which depends on the individual cluster history, cDE theories predict that the background $\Omega_{c} / \Omega_{b}$ ratio however increases with redshift. However, in top of that, the DM/baryon ratio measured in any virialized structure, exceeds the background ratio at the redshift where it is observed and is expected to exhibit significant variations in different systems, being smaller in larger systems, in average.

It must be however outlined that the final baryon/DM ratio, in any galaxy cluster at 
any redshift, even in the absence of any stripping effect, is expected to by smaller than the background $\Omega_{b} / \Omega_{c}$ ratio at that redshift. In this paper dedicated to a technical analysis of cDE mass functions we refrain from discussing this feature in further detail, although relating it with the apparent baryon shortage in clusters, (see, e.g., 18]) seems suggestive.

Furthermore, when cluster data are obtained through the hot gas behavior, a complex interplay between baryons and potential well is expected. Once again, however, the model used for PS-ST estimates seems unsuitable to provide quantitative predictions, but anomalies in the temperature-luminosity relations are expected.

A PS-ST analysis allows however to formulate further predictions, besides those concerning the $\Omega_{b} / \Omega_{c}$ ratio in galaxy clusters and in galaxies. They concern the cluster mass function and its evolution.

No large differences between models were ever found in the mass functions at $z=0$, because of DE nature: just a slight excess, $\sim 10 \%$, on the very large cluster scale, where observed clusters are a few units, was found in $\mathrm{dDE}$, in respect to a $\Lambda \mathrm{CDM}$ cosmology.

Discrepancies can be more relevant between $\Lambda \mathrm{CDM}$ and $\mathrm{cDE}$, where a shortage of larger clusters is predicted. For $\beta=0.20$, they are half of $\Lambda \mathrm{CDM}$ at $\sim 3 \cdot 10^{14} h^{-1} M_{\odot}$ and less than $20 \%$ above a few $10^{15} h^{-1} M_{\odot}$. Such strong shortage can be falsified by samples just slightly richer than those now available. For $\beta=0.05$ the shift is smaller, hardly reaching $20 \%$, but still in the direction opposite to dDE.

This is a widespread feature of cDE models: the discrepancy of $\mathrm{dDE}$ from $\Lambda \mathrm{CDM}$ is partially or totally erased even by a fairly small DM-DE coupling, and many cDE predictions lay on the opposite side of $\Lambda \mathrm{CDM}$, in respect to $\mathrm{dDE}$. Therefore, if a $\Lambda \mathrm{CDM}$ model (or any uncoupled model) is used to fit galaxy or cluster data arising in a cDE cosmology, we expect that cluster data may yield a smaller $\sigma_{8}$, in comparison to the one worked out from other data sets.

Turning to the evolutionary predictions, we expect an evolution faster than $\Lambda$ CDM for any coupling $\beta>0.1$, again the opposite of what we expect in uncoupled dDE.

Most quantitative results given in this paper are worked out by assuming that the scalar field self-interaction potential is SUGRA, with $\Lambda=100 \mathrm{GeV}$. For the sake of comparison, in a few plots, results obtained for a RP potential are also shown. It should be reminded that, while the former potential predicts a linear behavior consistent with observations, the latter one can be fitted with linear observables only for quite low values of $\Lambda$, much below 
the one considered here; we selected it just to provide a direct comparison tool, so allowing us to conclude that a different self-interacion potential may cause quantitative shifts up to some $10 \%$, but hardly affect our general conclusions.

Altogether, there can be no doubt that cDE cosmologies open new prespectives for the solution of those problems where baryon-DM segregation due to hydrodynamics is apparently insufficient to explain observed features. These problems may range from the shortage of galaxy satellites in the local group, to galactic disk formation, up to the $L$ vs $T$ relation in large clusters. Stating how coupling can affect these and similar questions, by using a PS-ST approach, is hard. This calls for detailed n-body and hydrodynamical simulations of cDE cosmologies.

\section{ACKNOWLEDGMENTS}

Luca Amendola, Andrea Macciò and Loris Colombo are gratefully thanked for their comments on this work.

[1] Wetterich C. 1988, Nucl.Phys.B 302, 668

[2] Ratra B. \& Peebles P.J.E., 1988, Phys.Rev.D 37, 3406; Peebles P.J.E. \& Ratra B., 1988, ApJ. Lett., 325, L17

[3] Peebles P.J.E \& Ratra B., 2003, Rev. Mod. Phys., 75, 559

[4] Amendola L., 1999, Phys. Rev. D60, 043501

[5] Amendola L. \& Quercellini C., 2003, Phys. Rev. D68, 023514

[6] Maccio' A. V., Quercellini C., Mainini R., Amendola L., Bonometto S. A., 2004 Phys. Rev. D69, 123516

[7] Mainini R., Bonometto S.A., 2004, Phys.Rev.Lett. 93, 121301; Mainini R., Colombo L. \& Bonometto S.A., 2005, ApJ 635, 691-705

[8] Press W.H. \& Schechter P., 1974, ApJ, 187, 425

[9] Sheth R.K. \& Tormen G., 1999 MNRAS, 308, 119; Sheth R.K. \& Tormen G., 2002 MNRAS 329, 61; Jenkins, A., Frenk C.S., White S.D.M., Colberg J.M., Cole S., Evrard A.E., Couchman H.M.P. \& Yoshida N., 2001, MNRAS, 321, 372 
[10] Brax, P. \& Martin, J., 1999, Phys.Lett., B468, 40; Brax, P. \& Martin, J., 2000, Phys.Rev. D61, 103502; Brax P., Martin J., Riazuelo A., 2000, Phys.Rev. D62, 103505

[11] Mainini R., 2005, PRD 72, 083514 (paper I)

[12] Amendola L., Phys. Rev. 2004, D69, 2004, 103524

[13] Lahav, O., Lilje, P.R., Primack, J.R. \& Rees, M., 1991, MNRAS, 251,128; Brian, G. \& Norman, M., 1998, ApJ, 495, 80

[14] Mainini R., Maccio' A. V., Bonometto S. A., 2003, New Astron., 8, 173 Mainini R., Maccio' A. V., Bonometto S. A., Klypin A., 2003, ApJ, 599, 24

[15] Wang L. \& Steinhardt P.J., 1998, ApJ, 508, 483; Lokas E. L., Bode P., Hoffman Y., 2004, MNRAS, 349, 595 Horellou C., Berge J., 2005, astro-ph/0504465 Nunes N. J., da Silva A. C., Aghanim N., 2005, astro-ph/0506043

[16] Nunes N. J.\& Mota D.F, 2005, astro-ph/0409481 Manera M. \& Mota D., 2005, astro-ph/0504519

[17] Solevi P., Mainini R., Bonometto S.A., Maccio’ A.V., Klypin A., Gottloeber S., 2006 MNRAS 366,1346

[18] Allen S.W., Schmidt R.W., Fabian A.C., 2001, MNRAS 328, 137, Ettori S., 2003, MNRAS 344, L13

[19] Klypin A., Macciò A., Mainini R. \& Bonometto S.A., 2003 ApJ 599, 31 


\section{Appendix 1. The newtonian regime}

In the presence of inhomogeneities, the metric can read

$$
d s^{2}=a^{2}(\tau)\left[-(1+2 \psi) d \tau^{2}+(1-2 \psi) d x_{i} d x^{i}\right]
$$

provided that no anisotropic stresses are considered, $\psi$ being the gravitational potential in the Newtonian gauge. Let us describe DE field fluctuations $\delta \phi$ through

$$
\varphi=(4 \pi / 3)^{1 / 2}\left(\delta \phi / m_{p}\right)
$$

and expand fluctuations in components of wavenumber $\mathbf{k}$; let also be $\lambda=\mathcal{H} / k$. Let then be

$$
f=\phi^{-1} \sqrt{3 / 16 \pi G} \ln \left(V / V_{o}\right), \quad f_{1}=\phi \frac{d f}{d \phi}+f, \quad f_{2}=\phi \frac{d f}{d \phi}+2 f+f_{1} ;
$$

$V_{o}$ being a reference value of the potential. It is also useful to define $Y^{2}=8 \pi G V(\phi) a^{2} / 3 \mathcal{H}^{2}$. The equations ruling the evolution of the $\varphi$ field and gravity, keeping just the lowest order terms in $\lambda$, as is needed to obtain their Newtonian limit, then read

$$
\begin{gathered}
\psi=-\frac{3}{2} \lambda^{2}\left(\Omega_{b} \delta_{b}+\Omega_{c} \delta_{c}+6 X \varphi+2 X \varphi^{\prime}-2 Y^{2} f_{1} \varphi\right), \quad \psi^{\prime}=3 x \varphi-\psi, \\
\varphi^{\prime \prime}+\left(2+\frac{\mathcal{H}^{\prime}}{\mathcal{H}}\right) \varphi^{\prime}+\lambda^{-2} \varphi-12 X \varphi+4 \psi X+2 Y^{2}\left(f_{2} \varphi-f_{1} \psi\right)=\beta \Omega_{c}\left(\delta_{c}+2 \psi\right) ;
\end{gathered}
$$

let us remind that $X$ is defined in eq. (8) and notice that, if DE kinetic (and/or potential) energy substantially contributes to the expansion source, $X$ (and/or $Y$ ) is $\mathcal{O}(1)$.

In the Newtonian limit, $\varphi$ derivatives shall be neglected, the oscillations of $\varphi$ and the potential term $f_{2} Y^{2} \varphi$ should be averaged out, by requiring that $\lambda<<\left(f_{2} Y\right)^{-1}$, and, in eq. (34), the metric potential $\psi\left(\propto \lambda^{2}\right)$ can also be neglected. Then, eqs. (33)-(34) become

$$
\psi=-\frac{3}{2} \lambda^{2}\left(\Omega_{b} \delta_{b}+\Omega_{c} \delta_{c}\right), \quad, \quad \lambda^{-2} \varphi \simeq \beta \Omega_{c} \delta_{c} .
$$

Baryon and DM density fluctuations are then ruled by the eqs. (6)-(7), derived from the stress-energy pseudo-conservation $T_{\nu ; \mu}^{\mu}=0$. Thereinside, ' yields differentiation with respect to $\ln a$. [Let us then notice that, taking $\Omega_{b}<<\Omega_{c}$, putting $\delta_{c} \propto e^{\int \mu(\alpha) d \alpha}$ and $\delta_{b}=b \delta_{c}$ with $b=$ cost, eqs. (6), allow us to obtain the bias factor (11)].

The acceleration of a DM or baryon particle of mass $m_{c, b}$ can then be derived from eqs. (6) - (77). Let it be in the void, at a distance $r$ from the origin, where a DM (or baryon) particle of mass $M_{c}$ (or $M_{b}$ ) is set, and let us remind that, while $\bar{\rho}_{b} \propto a^{-3}$, it is

$$
\bar{\rho}_{c}=\bar{\rho}_{o c} a^{-3} e^{-C\left(\phi-\phi_{0}\right)}, \quad \rho_{M_{c}}=M_{o c} a^{-3} e^{-C\left(\phi-\phi_{0}\right)} \delta(0)
$$


because of the DE-DM coupling (the subscript $o$ indicates values at the present time $\tau_{o}$; let also be $\left.a_{o}=1\right)$. We can then assign to each DM particle a varying mass $M_{c}(\phi)=$ $M_{o c} e^{-C\left(\phi-\phi_{0}\right)}$

Then, owing to eq. (36), and assuming that the density of the particle widely exceeds the background density, it is

$$
\Omega_{c} \delta_{c}=\frac{\rho_{M_{c}}-\bar{\rho}_{c}}{\rho_{c r}}=\frac{8 \pi G}{3 \mathcal{H}^{2} a} M_{c}(\phi) \delta(0), \quad \Omega_{b} \delta_{b}=\frac{\rho_{M_{b}}-\bar{\rho}_{b}}{\rho_{c r}}=\frac{8 \pi G}{3 \mathcal{H}^{2} a} M_{b} \delta(0),
$$

( $\rho_{c r}$ is the critical density and $\delta$ is the Dirac distribution). Reminding that $\nabla \cdot \mathbf{v}_{c, b}=\theta_{c, b} \mathcal{H}$ and using the ordinary (not conformal) time, eq. (6) yields

$$
\nabla \cdot \dot{\mathbf{v}}_{c}=-H(1-2 \beta X) \nabla \cdot \mathbf{v}_{c}-4 \pi G a^{-2}\left(\gamma M_{c}(\phi)+M_{b}\right) \delta(0)
$$

(dots yield differentiation in respect to ordinary time and $H=\dot{a} / a$ ). Taking into account that the acceleration is radial, as the attracting particles are in the origin, it will be

$$
\int d^{3} r \nabla \cdot \dot{\mathbf{v}}=4 \pi \int d r d\left(r^{2} \dot{v}\right) / d r=4 \pi r^{2} \dot{v}
$$

Accordingly, the radial acceleration of a DM particle is

$$
\dot{v}_{c}=-(1-2 \beta X) H \mathbf{v}_{c} \cdot \mathbf{n}-\frac{G^{*} M_{c}(\phi)}{R^{2}}-\frac{G M_{b}}{R^{2}}
$$

( $\mathbf{n}$ is a unit vector in the radial direction; $R=a r$ ).

By following a similar procedure for baryons, we obtain:

$$
\dot{v}_{b}=-H \mathbf{v}_{b} \cdot \mathbf{n}-\frac{G M_{c}(\phi)}{R^{2}}-\frac{G M_{b}}{R^{2}}
$$

In the presence of full spherical symmetry, $\mathbf{v}_{\mathbf{b}, \mathbf{c}} \cdot \mathbf{n}=v_{b, c}$; being then $b=r_{b}$ and $c=r_{c}$, eqs. (14) immediately follow.

For the sake of completeness, here below, we write also the equations for the physical DM and baryons radii which easily follow from eqs. (39) and (40). While for baryons we have the usual Friedman-like equations

$$
\ddot{R}_{b}^{n}=-\frac{4 \pi}{3} G\left[\rho_{c}+\rho_{b}+\rho_{D E}(1+3 w)\right] R_{b}^{n}
$$

for DM we have

$$
\ddot{R}_{c}^{n}=C \dot{\phi} \dot{R}_{c}^{n}-C \dot{\phi} H R_{c}^{n}-\frac{4 \pi}{3} G\left[\bar{\rho}_{c}+\gamma\left(\rho_{c}-\bar{\rho}_{c}\right)+\rho_{b}+\rho_{D E}(1+3 w)\right] R_{c}^{n} .
$$

